\title{
Intracardiac Lateral Tunnel Fontan by using Right Atrial Wall
}

\author{
Md Zahidul Islam¹, AM Asif Rahim², Kazi Abul Hasan ${ }^{1}$, Syed Imtiaz Ahsan ${ }^{1}$ \\ ${ }^{1}$ Department of Paediatric Cardiac Surgery, National Institute of Cardiovascular Diseases ,Dhaka, \\ ${ }^{2}$ Department of Cardiac Surgery, Chittagong Medical College Hospital, Chittagong
}

\begin{abstract}
Key Words : Congenital heart disease, transposition of great arteries, Lateral tunnel Fontan, Right atrial wall.
\end{abstract}

\begin{abstract}
The Fontan procedure is a palliative surgical procedure used in children with complex congenital heart defects. It involves diverting the venous blood from the right atrium to the pulmonary arteries without passing through the morphologic right ventricle. A 23 years old adult male from Noakhali admitted with the diagnosis of transposition of great arteries (D-TGA) with large perimembranous ventricular septal defect (PMVSD) with severe pulmonary stenosis (PS) and single ventricle morphology in National Institute of Cardiovascular Disease (NICVD), Dhaka. He underwent Intracardiac lateral tunnel Fontan by using right atrial wall. Patient recovered uneventfully in his early postoperative period. The lateral tunnel Fontan procedure results in excellent long-term outcome even when used in patients with diverse anatomic diagnoses. The incidence of atrial tachyarrhythmia is low and mainly depends on the underlying cardiac morphology and preoperative arrhythmia. The good long-term outcome after an intracardiac lateral tunnel Fontan procedure should serve as a basis for comparison with other surgical alternatives. But using right atrial wall as a buffle is rare and done first time in Bangladesh.
\end{abstract}

(Cardiovasc. j. 2016; 9(1): 68-72)

\section{Introduction}

The Fontan procedure is a palliative surgical procedure used in children with complex congenital heart defects. It involves diverting the venous blood from the right atrium to the pulmonary arteries without passing through the morphologic right ventricle; i.e., the systemic and pulmonary circulations are placed in series with the functional single ventricle. It was initially described in 1971 by Dr. Francois Marie Fontan from Bordeaux, France, and later in 1973 by Dr. Guillermo Kreutzer from Buenos Aires, Argentina separately as a surgical treatment for tricuspid atresia.The Fontan procedure is used in pediatric patients who possess only a single functional ventricle, either due to lack of a heart valve (e.g. tricuspid or mitral atresia), an abnormality of the pumping ability of the heart (e.g. hypoplastic left heart syndrome or hypoplastic right heart syndrome), or a complex congenital heart disease where a bi-ventricular repair is impossible or inadvisable. The single ventricle is doing nearly twice the expected amount of work because it has to pump blood for the body and lungs. Patients typically present as neonates with cyanosis or congestive heart failure. Fontan completion is usually carried out when the patient is 2-5 years of age, but is also performed before 2 years of age. ${ }^{1-3}$

There are three variations of the Fontan procedure 1) Atrio-pulmonary connection (the original technique), 2) Intracardiac total cavopulmonary connection (lateral tunnel) and 3) Extracardiac total cavopulmonary connection. The Fontan is usually done as a two staged repair. The first stage, also called a Bidirectional Glenn procedure or HemiFontan (Kawashima procedure), involves redirecting oxygen-poor blood from the top of the body to the lungs. That is, the pulmonary arteries are disconnected from their existing blood supply (e.g. a shunt created during a Norwood procedure, a patent ductus arteriosus, etc.). The superior vena cava (SVC), which carries blood returning from the upper body, is disconnected from the heart and instead redirected into the pulmonary arteries. The inferior vena cava (IVC), which carries blood returning from the lower body, continues to connect to the heart.The second stage, also called Fontan completion, involves redirecting the blood from the IVC to the lungs. At this point, the oxygenpoor blood from upper and lower body flows through the lungs without being pumped (driven only by the pressure that builds up in the veins).

Address of Correspondence: Dr. Md. Zahidul Islam, Department of Paediatric Cardiac Surgery, NICVD,Dhaka, Bangladesh. Email- 
This corrects the hypoxia and leaves the single ventricle responsible only for supplying blood to the body. ${ }^{2,4}$

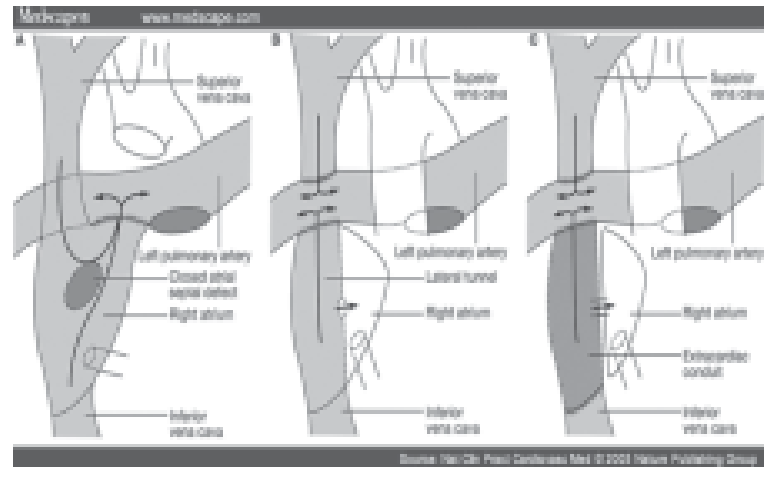

\section{Case report:}

A 23 years old adult young male admitted with bluish discoloration of whole body more prominent
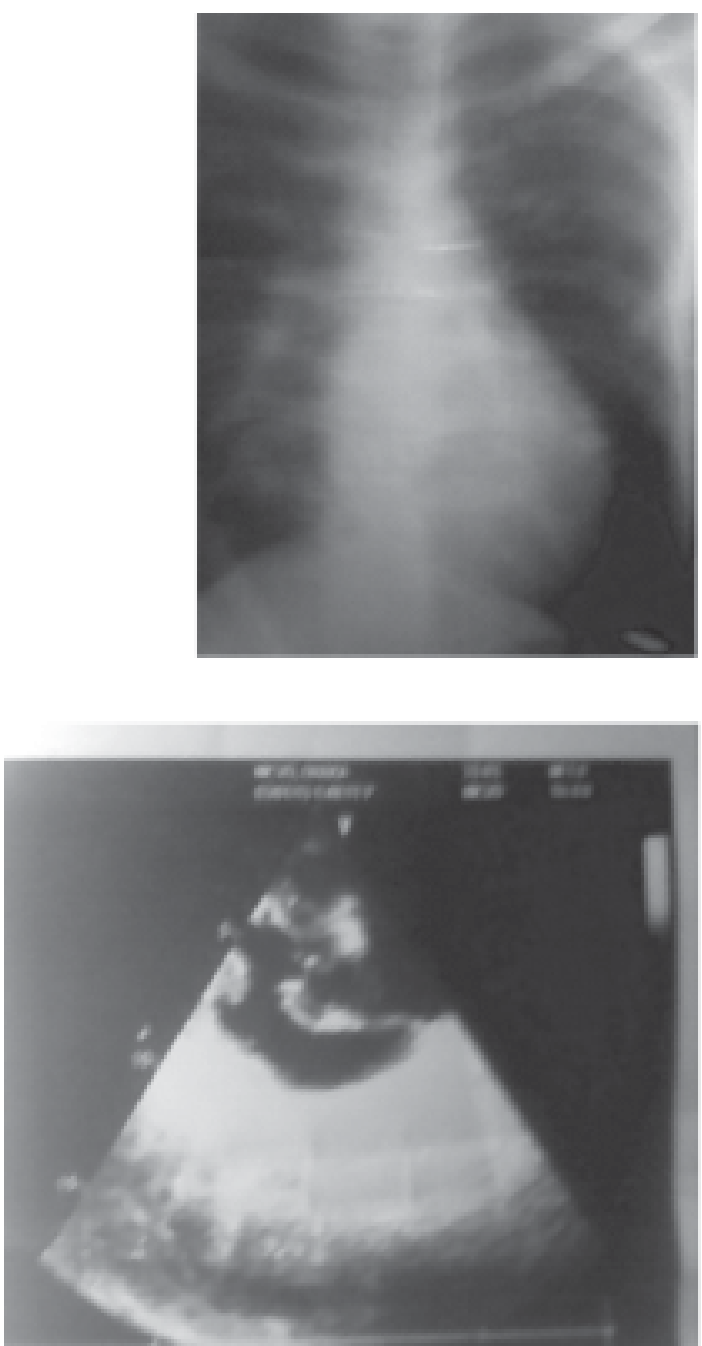

in nail, lips, nose and ear lobule since birth along with repeated bouts of respiratory tract infection. He also complaints of progressive breathlessness that increases in severity for last few years and become more severe for last 6 months with mild exertion. On examination his body build was below average, small for age, decubitus on choice, cyanosis and clubbing were present, neck vein not engorged and there were no leg odema or ascites. Pulse was $120 \mathrm{~b} / \mathrm{min}$, regular, blood pressure-90/60 mm of hg. respiratory rate $22 / \mathrm{min}$. On palpation apex beat is slightly shifted laterally about $1 \mathrm{~cm}$ from midclavicular line. On auscaltation first and second heart sounds were normal, there were a pansystolic murmur along left $3^{\text {rd }}$ or $4^{\text {th }}$ space with no radiaion. Chest X-ray P/A view revealed enlarged cardiac shadow with congested lungs more
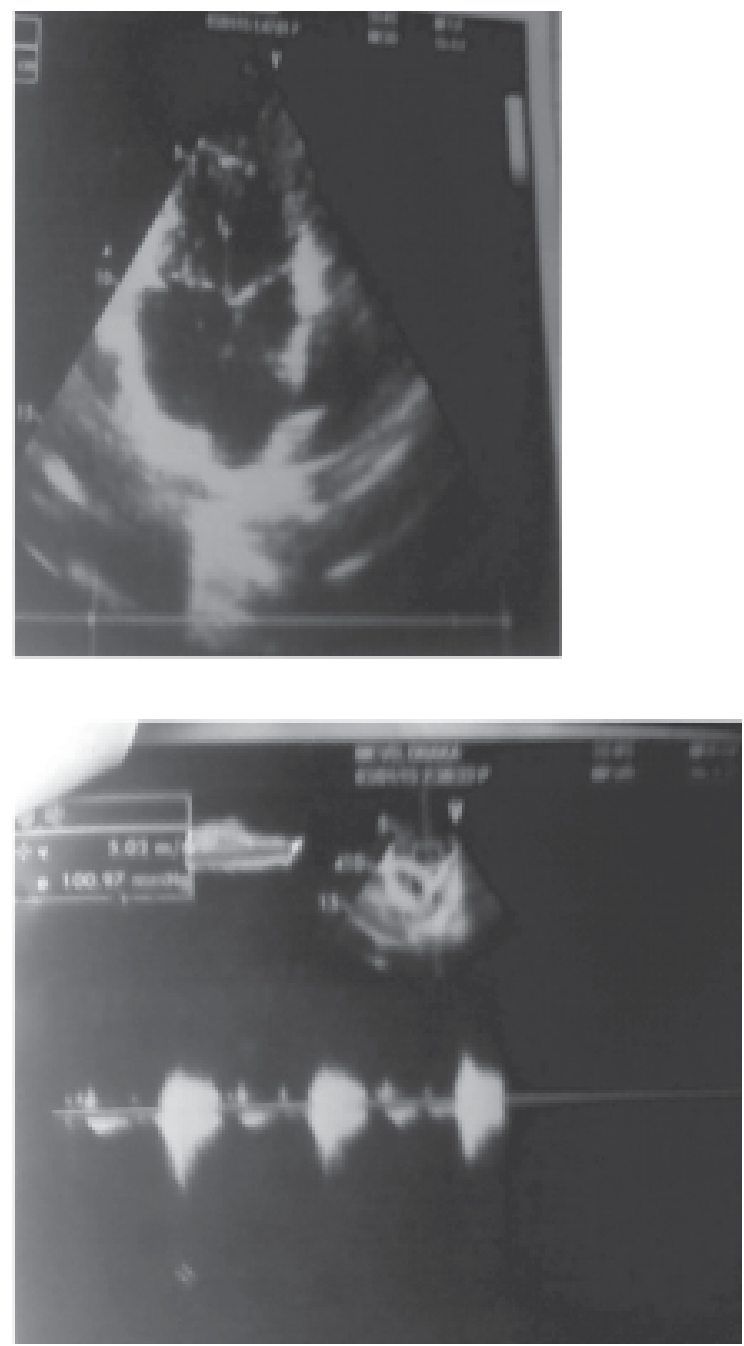

Fig.-1: Preoperative chest X-ray and Echocardiography. 


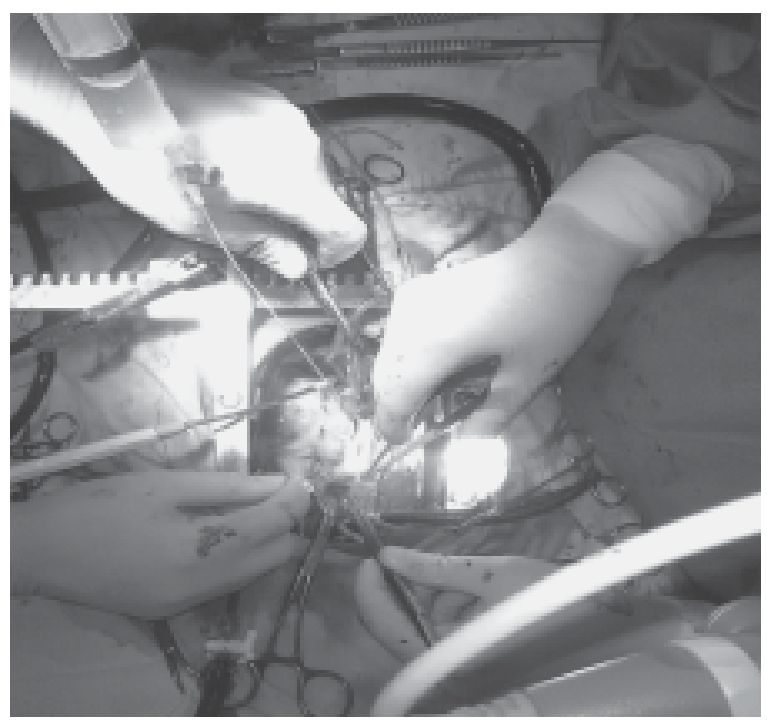

Fig.-2: Peroperative picture.
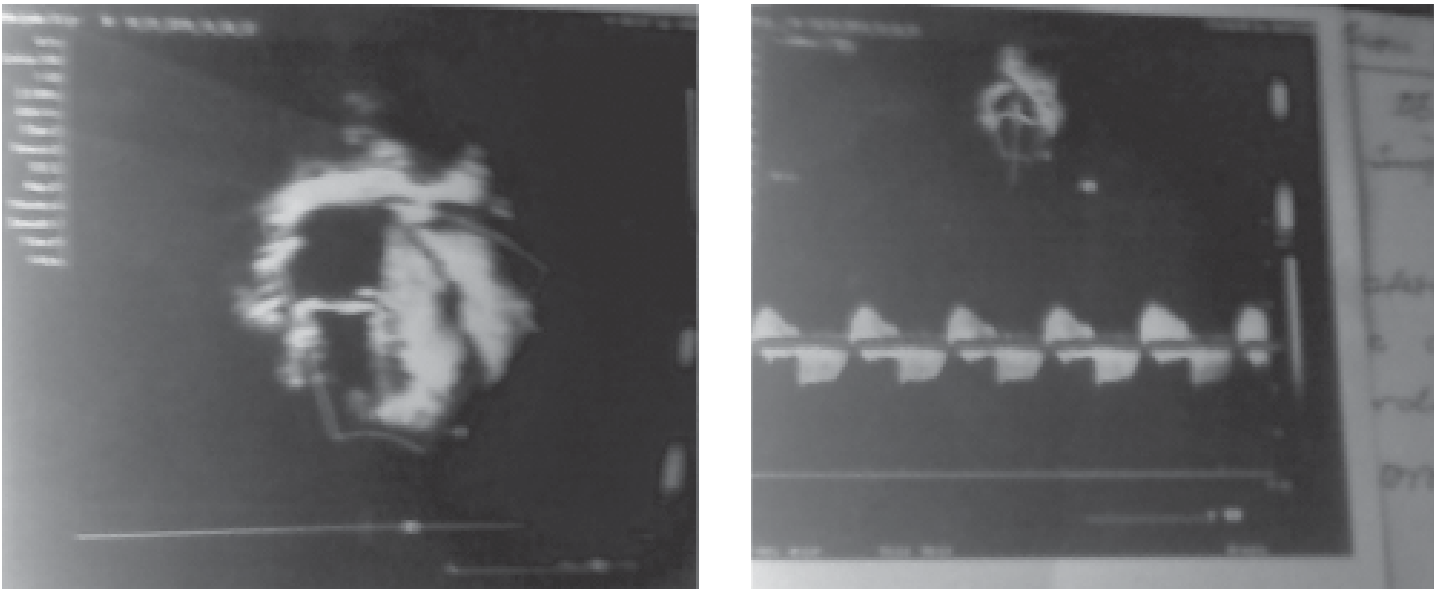

Fig.-3: Postoperative Echocardiography at $10^{\text {th }}$ POD.

prominent in hilar region. Echocardiography revealed D-TGA. PM VSD, severe PS and single ventricle. CT pulmonary angiogram revealed severely stenosed pulmonary infundibulum and valve although main pulmonary artery (MPA), right pulmonary artery (RPA), left pulmonary artery (LPA), Aorta, SVC, IVC were of normal size with no evidence of other congenital anomalies. In Heart there were three chambered heart with single ventricle. Both the inlets were normal. But double outlets single ventricle was detected with large atrial septal defect (ASD) secondum. After detail counseling patient underwent open heart surgery. A median sternotomy was performed in all patients. The thymus gland was removed completely and pericardium was opened. The right and left superior vena cavae were dissected and mobilized upto the inominate vein. Also the right and left pulmonary arteries were dissected and mobilized. Azygos vein was doubly ligated and divided.Though intra-cardiac or lateral tunnel method is the 'older' method (introduced in 1987) and the extra-cardiac method was more recently developed (1990); we like to perform the intracardiac method as our preferred option. The lateral tunnel/intra-cardiac method involves sewing a piece of plastic inside the right atrium to route all blood from the lower part of the body but here in this case we use patient's own right atrial wall instead of PTFE tube (via the inferior vena cava) 
to the lungs and thereby performed intracardiac lateral tunnel Fontan by using patient's own right Arial wall. Patient recovered uneventfully and discharged on $10^{\text {th }}$ Post operative day.

\section{Discussion:}

After Fontan completion, blood must flow through the lungs without being pumped by the heart. Therefore, children with high pulmonary vascular resistance may not tolerate a Fontan procedure. Often, cardiac catheterization is performed to check the resistance before proceeding with the surgery. This is also the reason a Fontan procedure cannot be done immediately after birth; the pulmonary vascular resistance is high in utero and takes months to drop. ${ }^{2}$

Regarding post operative complication In the short term, children can have trouble with pleural effusions (fluid building up around the lungs). This can require a longer stay in the hospital for drainage with chest tubes. To address this risk, some surgeons make a fenestration from the venous circulation into the atrium. When the pressure in the veins is high, some of the oxygenpoor blood can escape through the fenestration to relieve the pressure. However, this results in hypoxia, so the fenestration may eventually need to be closed by an interventional cardiologist.In the long term postoperative complication, children can have trouble with atrial flutter and atrial fibrillation because of scarring in the atrium, especially if the connection of the IVC to pulmonary arteries involved an Intracardiac baffle (instead of an extracardiac conduit). This sometimes requires treatment such as radiofrequency ablation. Other long-term risks include protein-losing enteropathy and chronic renal insufficiency, although understanding of these risks is still incomplete. Some patients require long-term anticoagulant. Four case reports of hepatocellular carcinoma following the Fontan procedure were reported by physicians at the Mayo Clinic. This group of patients may warrant screening for this malignancy, especially if the alpha-fetoprotein level is elevated. The Fontan procedure is palliative not curative - but in many cases it can result in normal or near-normal growth, development, exercise tolerance and good quality of life. ${ }^{7}$ In 20 $30 \%$ cases, patients will eventually require heart transplantation. ${ }^{4}$
The lateral tunnel or intra-cardiac method requires placing a piece of plastic ("baffle") inside of the atrium. The lateral tunnel/intra-cardiac method has had good early, medium and long-term outcomes as well in previous follow-up studies. However, this requires sewing the piece of plastic (Gore Tex/PTFE tube graft) or right atrial wall (we use here) inside the heart, which may increase the risk of atrial arrhythmias. The extra-cardiac method avoids having to sew this piece of plastic inside of the heart and so a theoretical advantage is that this method may decrease risk of future heart rhythm issues. However, note that this generally requires that the surgery be performed later in life because you are placing a piece of plastic in the heart that will not grow with the patient. Another possible advantage of the extra-cardiac method is that it can allow surgeons to perform the procedure without aortic cross-clamping (where they prevent the blood from leaving the heart) and even without cardiopulmonary bypass, which may have some advantages for short- and long-term outcomes. Some study revealed, that aortic cross clamp always used for the lateral tunnel/intracardiac method but used in $51 \%$ of patients for the extra-cardiac method. No difference in time on cardiopulmonary bypass between intra-cardiac and extra-cardiac method but patients undergoing intracardiac method were on aortic cross clamping longer than those using extra-cardiac method. Study showed Patients undergoing the lateral tunnel/intra-cardiac method on bypass (mean $=134$ min) nearly the same amount of time as those undergoing the extra-cardiac method (mean $=145$ $\min$ ). However, $100 \%$ of patients undergoing the lateral tunnel/intra-cardiac method had aortic cross-clamping and for a longer period of time $($ mean $=55 \mathrm{~min})$ than those that had the extracardiac method ( $52 \%$ of patients, mean $=26$ $\mathrm{min}$ ). No difference in time on ventilator, time in intensive care unit, duration of chest tube drainage, and hospital stay between those having the lateral tunnel/intra-cardiac method vs. those undergoing the extra-cardiac method. This is an interesting result given that the lateral tunnel/intra-cardiac method is theoretically supposed to improve shortterm outcome (that soon after the surgery) because of decreased chest tube drainage, etc. However, here they didn't find any differences between the two methods. No difference in type or frequency 
of medications given to patients that underwent intra-cardiac vs. extra-cardiac Fontan. Though this probably attributable to the hospital itself and how they treat their patients, most of the patients were on asprin (94\%) and there were no other differences between the type of frequency of medications taken between patients that underwent intra-cardiac or extra-cardiac method. No difference in heart rhythm problems between patients that had undergone intra-cardiac vs. extra-cardiac Fontan at 3 years after the surgery. This is somewhat surprising that $15 \%$ of patients that had undergone the intra-cardiac Fontan had heart rhythm issues (sinus node dysfunction) whereas more (28\%) patients that had underwent the extra-cardiac Fontan had heart rhythm issues. Although this is not statistically different, this is opposite than what would be expected. Two patients underwent permanent pacemaker implantation (1 lateral tunnel and 1 extra-cardiac method) and in one case for slow junctional rhythm. No difference in the post-operative blood pressure in various parts of the atrium and in the Fontan pathway ("Fontan pressure" and transpulmonary gradient) between patients that underwent the intra-cardiac vs. extracardiac Fontan. The authors provide brief discussion how these pressures can be predictive of early Fontan failure but they found no difference between these two methods in the first 24 hours after the Fontan. ${ }^{2,4}$

Kaplan-Meier estimated survival was $93 \%$ at 5 years and $91 \%$ at 10 years, and freedom from failure was $90 \%$ at 5 years and $87 \%$ at 10 years. Freedom from new supraventricular tachyarrhythmia was $96 \%$ at 5 years and $91 \%$ at 10 years; freedom from new bradyarrhythmia was $88 \%$ at 5 years and $79 \%$ at 10 years and ultimately high rates of early survival 3-5 years after the Fontan for both the intra-cardiac (97\%) and extra-cardiac (91\%) methods. $^{2,4}$

\section{Conclusion:}

The lateral tunnel Fontan procedure results in excellent long-term outcome even when used in patients with diverse anatomic diagnoses. The incidence of atrial tachyarrhythmia is low and mainly depends on the underlying cardiac morphology and preoperative arrhythmia. The good long-term outcome after an intracardiac lateral tunnel Fontan procedure should serve as a basis for comparison with other surgical alternatives. Here we use patient's own right atrial wall instead of Gortex tube which is completely new concept in our situation and no such evidence based study on use of right atrial wall in intracardiac lateral tunnel Fontan has been performed so far. Our patient recovered uneventfully so this may be an acceptable procedure in near future in this operation instead of using Gortex tube.

\section{Conflict of Interest - None.}

\section{Refferences:}

1. Behrman, Richard E.; Robert M. Kliegman; Hal B. Jenson (2004). Nelson Textbook of Pediatrics(17th ed.). Saunders. ISBN 0-7216-9556-6.

2. Hirsch JC, Goldberg C, Bove EL; et al. Fontan operation in the current era: a 15-year single institution experience. Annals of Surgery 2008;248: 402-410.

3. Leval, Marc $R$ de. The Fontan circulation: a challenge to William Harvey?". Nature Clinical Practice Cardiovascular Medicine 2005;2 (4): 202-208.

4. Stamm C, Friehs I, Mayer JE Jr, Zurakowski D, Triedman JK, Moran AM, et al. Long-term results of the lateral tunnel Fontan operation. $J$ ThoracCardiovasc Surg Jan2001;121(1):28-41. 